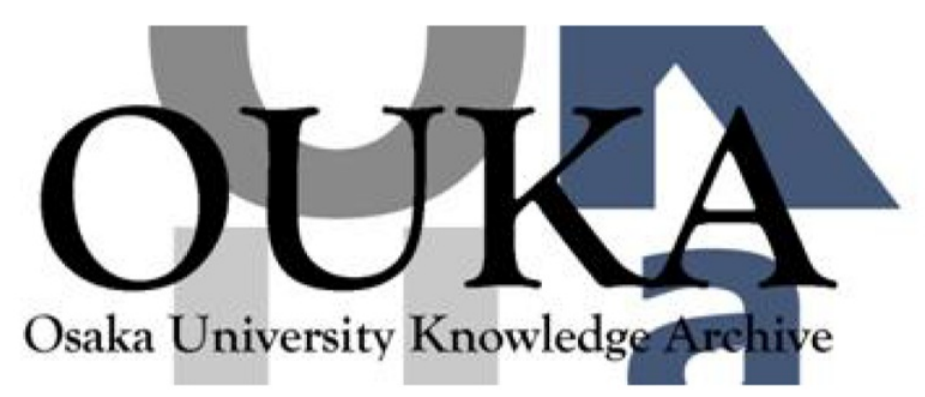

\begin{tabular}{|c|l|}
\hline Title & $\begin{array}{l}\text { Electro-optical response of reflected light } \\
\text { from freely suspended smectic liquid-crystal } \\
\text { fi lms }\end{array}$ \\
\hline Author(s) & $\begin{array}{l}\text { Nakano, Kazuyuki; Ozaki, Masanori; Yoshino, } \\
\text { Katsumi }\end{array}$ \\
\hline Citation & $\begin{array}{l}\text { Journal of Applied Physics. 92(11) p. 6384- } \\
\text { p. 6389 }\end{array}$ \\
\hline Issue Date & $2002-11-02$ \\
\hline oaire:version & VoR \\
\hline URL & https://hdl.handle.net/11094/75851 \\
\hline rights & \\
\hline Note & \\
\hline
\end{tabular}

Osaka University Knowledge Archive : OUKA

https://ir. Library. osaka-u. ac. jp/

Osaka University 


\section{Electro-optical response of reflected light from freely suspended smectic liquid-crystal films}

Cite as: Journal of Applied Physics 92, 6384 (2002); https://doi.org/10.1063/1.1518761

Submitted: 25 June 2002 . Accepted: 10 September 2002 . Published Online: 12 November 2002

Kazuyuki Nakano, Masanori Ozaki, and Katsumi Yoshino

View Online

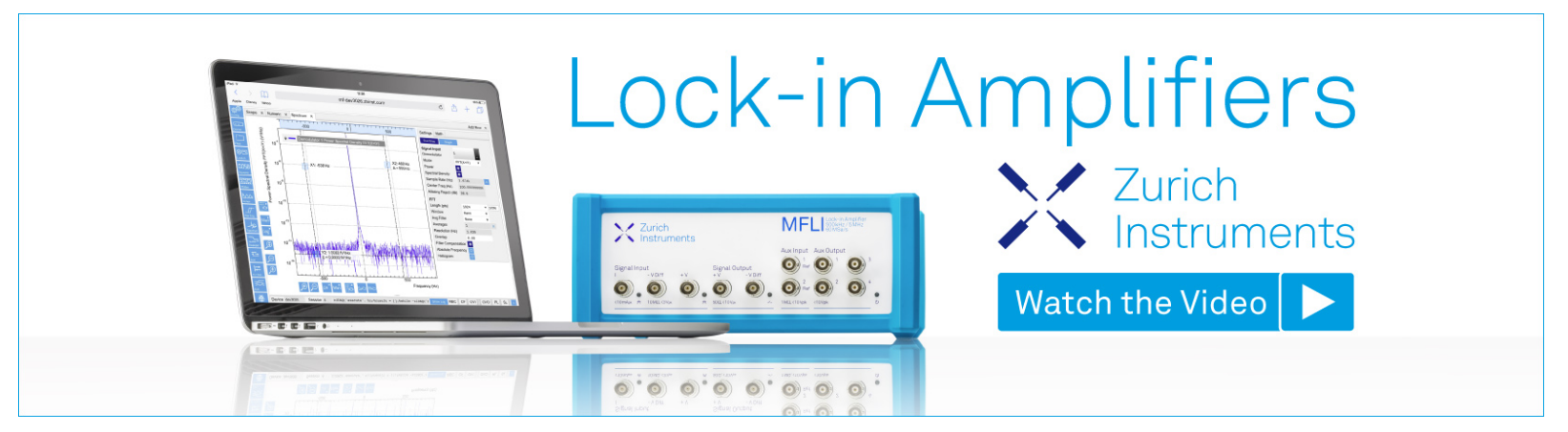




\title{
Electro-optical response of reflected light from freely suspended smectic liquid-crystal films
}

\author{
Kazuyuki Nakano, Masanori Ozaki, a) and Katsumi Yoshino \\ Department of Electronic Engineering, Graduate School of Engineering, Osaka University, 2-1 Yamada-oka, \\ Suita, Osaka 565-0871, Japan
}

(Received 25 June 2002; accepted 10 September 2002)

\begin{abstract}
We have studied the electro-optical response of reflected light from the surface of freely suspended liquid-crystal films (FSFs) in SmA, SmC*, and $\mathrm{SmC}_{\mathrm{A}}^{*}$ phases. Upon application of an electric field parallel to FSFs, the film vibrational spectrum generally contains a second-harmonic component of the applied frequency. In the SmA phase, only a second-harmonic component appeared in the fast Fourier transform (FFT) spectra of the reflected light. In the SmC* phase, however, many other components also appeared in the FFT spectra of the reflected light. These complicated characteristics observed in the ferroelectric phase might be attributed to the contribution of the transient scattering mode. On the other hand, in the $\mathrm{SmC}_{\mathrm{A}}^{*}$ phase, only the second-harmonic component appeared, similar to the case of the SmA phase. In the electric field high enough to cause an antiferroelectric-ferroelectric phase transition, however, many other components appear in the spectrum, in the same manner as that in the SmC* phase. (c) 2002 American Institute of Physics.
\end{abstract} [DOI: $10.1063 / 1.1518761]$

\section{INTRODUCTION}

The smectic liquid crystal has a layered structure and can be used in a freely suspended geometry, the thickness of which can be varied from two to several thousands of smectic layers. The freely suspended liquid crystal film (FSF) has attracted considerable attention as a two-dimensional system. ${ }^{1-4}$ This film is not influenced by substrate potentials and provides the unique possibility of studying the influence of reduced dimensionality on various physical properties of confined ordered liquids. ${ }^{5-7}$ In addition, it is sensitive to the external field and can be easily deformed upon application of external stress such as the acoustic vibration of air. ${ }^{8}$ Application of electric field can induce mechanical vibration in the FSF. This phenomenon due to the coupling of the electric field and the mechanical stress is called the electromechanical effect. In previous studies on the electromechanical effect, a sandwich cell geometry consisting of two glass substrates was used. ${ }^{9}$ However, the FSF can easily change shape by application of a small electric field, ${ }^{10,11}$ which is optimal geometry for measurement of the electromechanical effect.

In this article, we report the detailed characteristics of FSF vibration and molecular dynamics excited by the electric field in the $\mathrm{SmA}, \mathrm{SmC}^{*}$, and $\mathrm{SmC}_{\mathrm{A}}^{*}$ phases.

\section{EXPERIMENT}

The smectic liquid crystals used in this study were CS-1029 (Chisso), 4-(1-methyl-heptyloxycarbonyl-phenyl) $4^{\prime}$ - octylbiphenyl-4-carboxylate (MHPOBC) and 4-hexyloxyphenyl 4-octyloxybenzoate (HOPOOB), as shown in Fig. 1. The FSF was prepared in a rectangular hole $(2 \mathrm{~mm} \times 10 \mathrm{~mm})$ on a glass plate $(30 \mathrm{~mm} \times 30 \mathrm{~mm})$ in the smectic A (SmA) phase of CS-1029 and MHPOBC or the

\footnotetext{
a)Electronic mail: ozaki@ele.eng.osaka-u.ac.jp
}

smectic C (SmC) phase of HOPOOB, as shown in Fig. 2(a). Subsequently, the film was left undisturbed for several hours to obtain a uniform thickness of the film. The thickness of the FSF was determined by measuring the reflection spectrum of the film in the SmA phase (CS-1029 and MHPOBC) or the SmC phase (HOPOOB) using a photomultichannel analyzer (PMA-11, Hamamatsu). ${ }^{3,12}$

The experimental setup is presented in Fig. 2(b). For excitation of the vibration modes, alternating voltage $(1 \mathrm{kHz})$ was applied across the electrodes. The light source was a $\mathrm{He}-\mathrm{Ne}$ laser $(632.8 \mathrm{~nm})$. The laser beam was polarized parallel to the electric field ( $s$ polarization) by a polarizer. The reflected laser beam from the FSF surface was detected by a photodiode via an aperture used as a position-sensitive detector. The vibration spectrum was observed as a secondharmonic component of the applied frequency. The fast Fourier transform (FFT) spectra of the reflected light were measured by a digital phosphor oscilloscope (TDS3012, Tektronix).

\section{RESULTS}

Figure 3 shows FFT spectra of the reflected light upon applying sinusoidal voltage at $1 \mathrm{kHz}$ frequency in the $\mathrm{SmA}$ $\left(140{ }^{\circ} \mathrm{C}\right), \mathrm{SmC}^{*}\left(120^{\circ} \mathrm{C}\right)$, and $\mathrm{SmC}_{\mathrm{A}}^{*}\left(100{ }^{\circ} \mathrm{C}\right)$ phases of MHPOBC. The film thickness is 42 layers. As evident from Fig. 3, the spectrum strongly depends on the phases, namely, in the SmA phase, only one signal was observed at $2 \mathrm{kHz}$, which corresponds to a second-harmonic component of the applied voltage. In contrast, in the $\mathrm{SmC}^{*}$ phase, not only the second-harmonic but also fundamental and higher-order components of the signal appear in the spectrum. In the $\mathrm{SmC}_{\mathrm{A}}^{*}$ phase, fundamental and second-harmonic components are mainly observed, which higher-order ones do not appear in the FFT spectrum. 
CS-1029

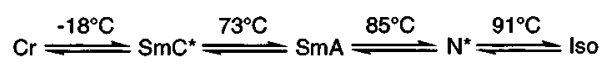

MHPOBC

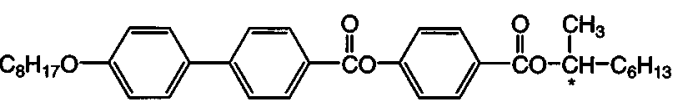

4-(1-methyl-heptyloxycarbonyl-phenyl) 4'-octylbiphenyl-4-carboxylate $\mathrm{Cr} \stackrel{30^{\circ} \mathrm{C}}{\rightleftharpoons} \mathrm{Sml}_{\mathrm{A}}^{*} \stackrel{65^{\circ} \mathrm{C}}{\rightleftharpoons} \mathrm{SmC}_{\mathrm{A}^{*}} \stackrel{118.3^{\circ} \mathrm{C}}{=} \mathrm{SmC}_{\gamma} \stackrel{119^{\circ} \mathrm{C}}{=} \mathrm{SmC} \stackrel{122^{\circ} \mathrm{C}}{\rightleftharpoons} \mathrm{SmA} \rightleftharpoons 156^{\circ} \mathrm{C}$ Iso

HOPOOB

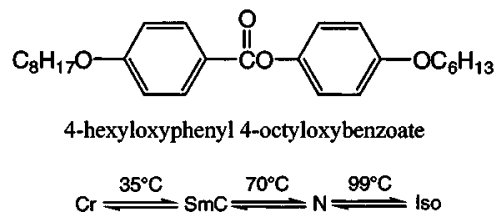

FIG. 1. Molecular structures and phase sequences of CS-1029, MHРOBC, and HOPOOB.

Figure 4 shows the electric field dependence of the signal intensity of each component in the FFT spectra shown in Fig. 3. In the SmA phase, as evident from Fig. 4(a), only the second-harmonic component is observed in the entire voltage range and its signal intensity is proportional to the square of electric field $E$. On the other hand, in the $\mathrm{SmC}^{*}$ phase, besides the second-harmonic component, fundamental and other higher-order components are observed. Although, in the $\mathrm{SmC}^{*}$ phase of this material, there is a large amount of scatter in the measured values, for a chirally doped HOPOOB, the field dependence of the signal in the SmC*

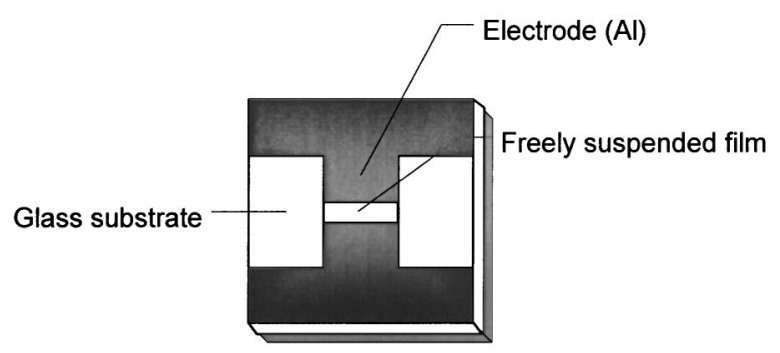

(a)

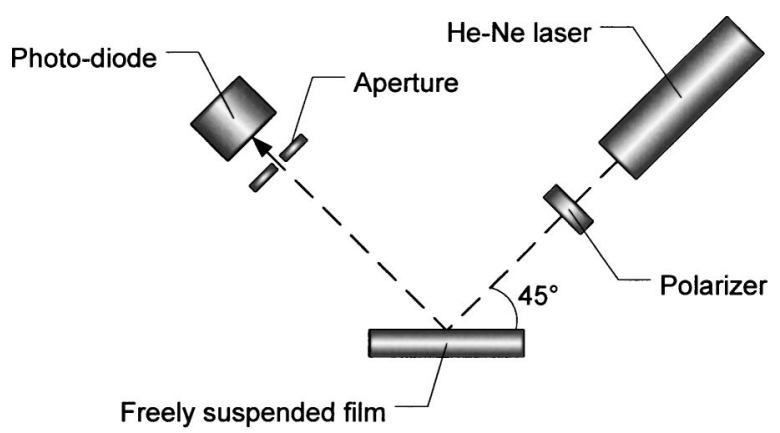

(b)

FIG. 2. Schematic diagram of measurement of film vibration by reflected light.

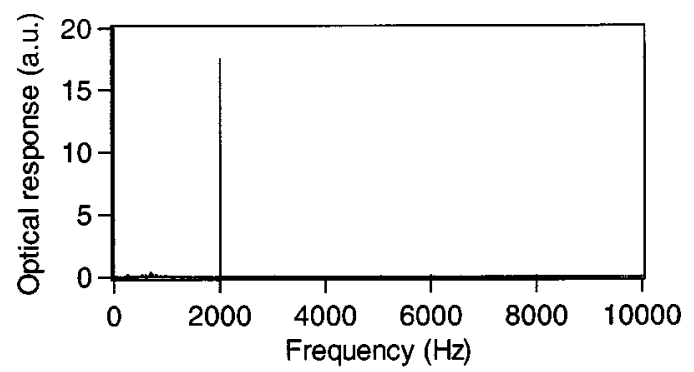

(a)

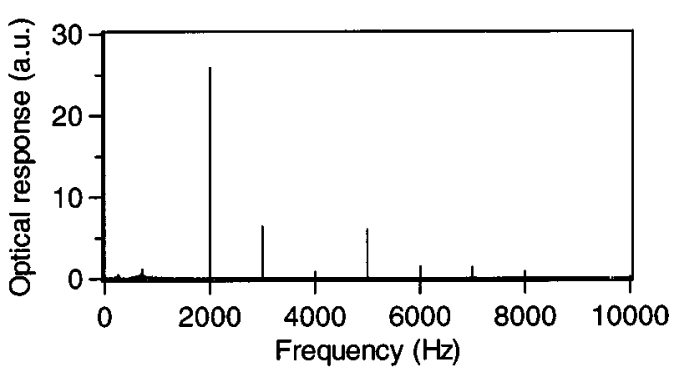

(b)

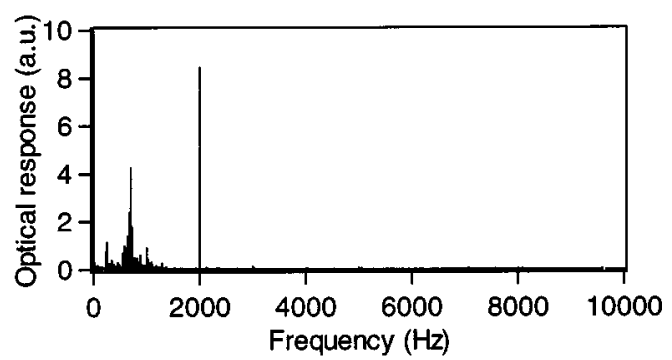

(c)

FIG. 3. FFT spectra of the reflected light upon applying a sinusoidal voltage at $1 \mathrm{kHz}$ frequency of MHPOBC; (a) $\mathrm{SmA}$, (b) $\mathrm{SmC}^{*}$, and (c) $\mathrm{SmC}_{\mathrm{A}}^{*}$.

phase follows the power laws corresponding to each order, as shown in Fig. 6(b). In the $\mathrm{SmC}_{\mathrm{A}}^{*}$ phase, as shown in Fig. 4(c), only fundamental and second-harmonic components are observed at a small electric field. At higher field ( $>5 \mathrm{kV} / \mathrm{cm}$ ), however, a third-harmonic component appears in the same manner as in the $\mathrm{SmC}^{*}$ phase, as shown in Fig. 4(c). This might be associated with the field-induced transition from the antiferroelectric to ferroelectric phases at about $5 \mathrm{kV} / \mathrm{cm}$.

From these results, it is found that the higher-order components were related to the molecular tilt and ferroelectricity.

To clarify the influence of the ferroelectricity on the tilted smectic phase, we compared the field (sine wave, 1 $\mathrm{kHz}$ ) dependence of the FFT spectrum of the reflected light in the SmC phase (HOPOOB) with that in the $\mathrm{SmC}^{*}$ phase [HOPOOB doped with a chiral molecule (S811, Mark) of 5 wt $\%$ concentration], as shown in Fig. 5. The temperature was $45^{\circ} \mathrm{C}$ in both phases. The film thicknesses are 74 and 105 layers for the $\mathrm{SmC}$ and $\mathrm{SmC}^{*}$ films, respectively. Figures 6(a) and 6(b) show electric field dependences of the optical response of each component of the FFT spectra in the $\mathrm{SmC}$ and $\mathrm{SmC}^{*}$ phases, respectively. As evident from Figs. 6(a) and 6(b), only the second-harmonic component was observed in the SmC phase. In contrast, fundamental and 


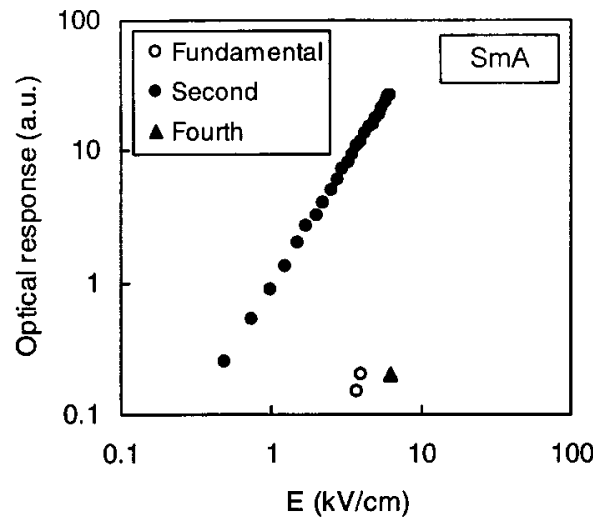

(a)

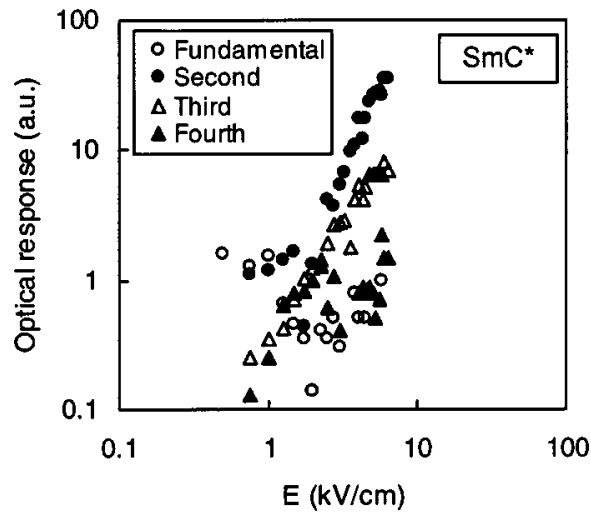

(b)

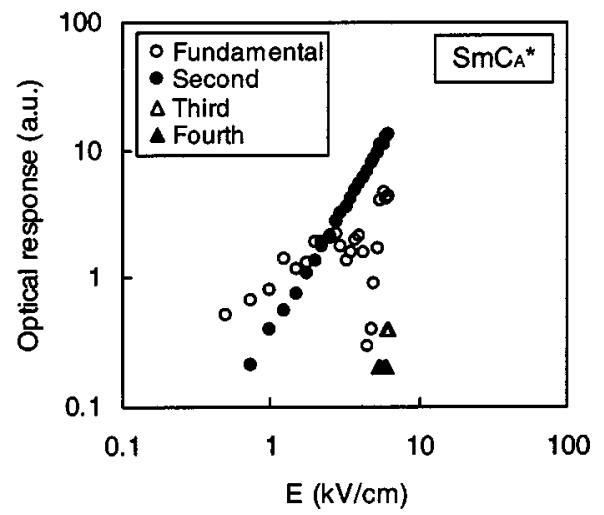

(c)

FIG. 4. Electric field dependence of the signal intensity of each component in the FFT spectra of MHPOBC; (a) SmA, (b) $\mathrm{SmC}^{*}$, and (c) $\mathrm{SmC}_{\mathrm{A}}^{*}$.

higher-order components also appeared in the $\mathrm{SmC}^{*}$ phase. From these results, it is considered that the higher-order components are peculiar to the $\mathrm{SmC}^{*}$ phase having ferroelectricity. In other words, the multicomponents in the FFT spectrum in the $\mathrm{SmC}^{*}$ phase may be related to a molecular reorientation based on the reversal of spontaneous polarization.

\section{DISCUSSION}

\section{A. SmA phase}

For the optical response measurement performed in this study, a small aperture is placed in front of the photodiode.

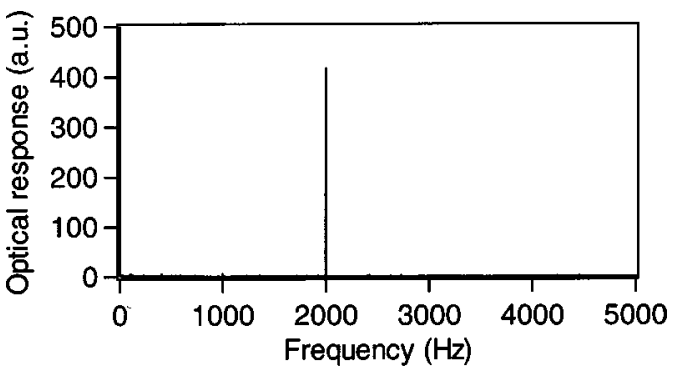

(a)

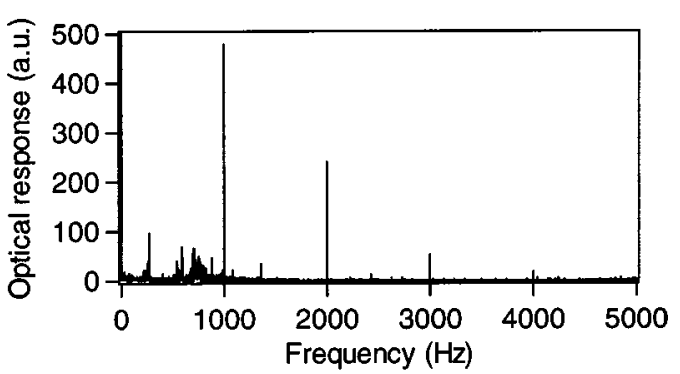

(b)

FIG. 5. FFT spectra of the reflected light intensity upon applying a sinusoidal voltage at $1 \mathrm{kHz}$ frequency of $\mathrm{HOPOOB}$; (a) SmC, and (b) SmC*.

In such reduced-aperture geometry, the intensity change in the reflected light from the FSF surface originates from three mechanisms, a reflectance change, a deflection of the reflected light beam, and scattering.

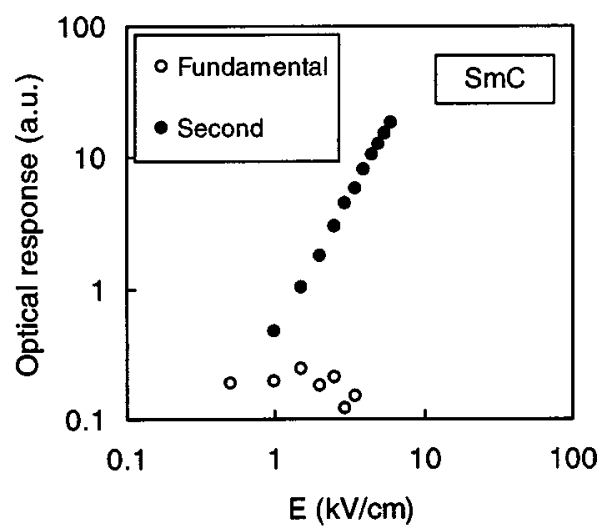

(a)

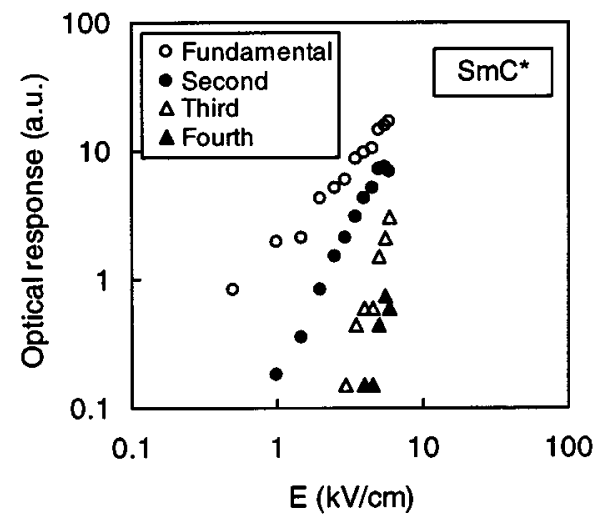

(b)

FIG. 6. Electric field dependence of the signal intensity of each component in the FFT spectra of HOPOOB; (a) SmC, and (b) $\mathrm{SmC}^{*}$. 


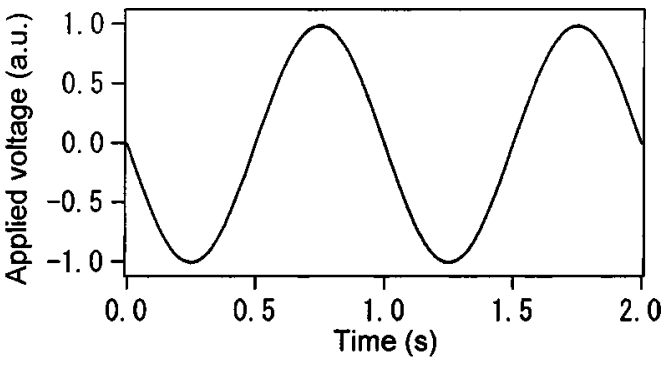

(a)

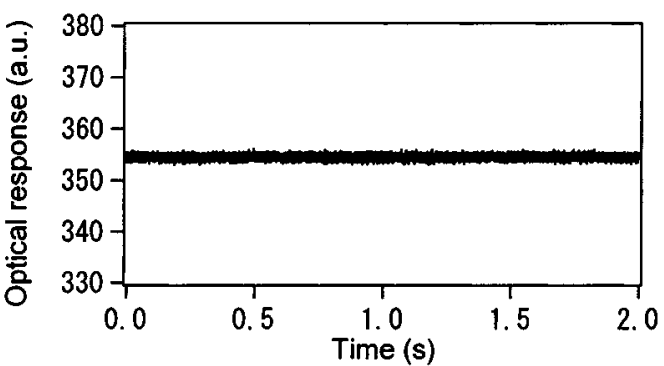

(b)

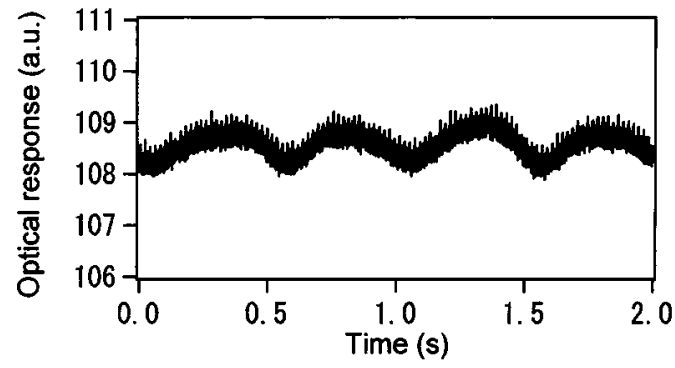

(c)

FIG. 7. Optical response to the sinusoidal wave form of the applied voltage in an open-aperture geometry of MHPOBC in the SmA phase; (a) applied voltage, (b) $s$ polarization, and (c) $p$ polarization.

First, in order to clarify the origin of the secondharmonic component observed in the SmA phase, the optical response measurement using an open-aperture geometry was also carried out. For the open-aperture geometry, no optical response signal is observed, although a clear secondharmonic peak appears in the reduced-aperture geometry, as shown in Fig. 3(a). This result indicates that the optical response in the SmA phase may be attributed to the beam deflection of the light from the FSF surface, which originates from the film vibration excited by the field application. Although two mechanisms for the optical response, i.e., reflectance change and scattering, are excluded according to this result, the following consideration also eliminates the reflectance change from the mechanism for the optical response in the SmA phase.

Figure 7 shows the optical response to the sinusoidal wave form of the applied voltage in an open-aperture geometry of MHPOBC at $s$ and $p$ polarization. Although the intensity of the reflected light hardly changed when light with $s$ polarization was irradiated, as shown in Fig. 7(b), the reflected wave form that corresponds to twice the applied frequency was observed when light with $p$ polarization was irradiated, as shown in Fig. 7(c). It is considered that the

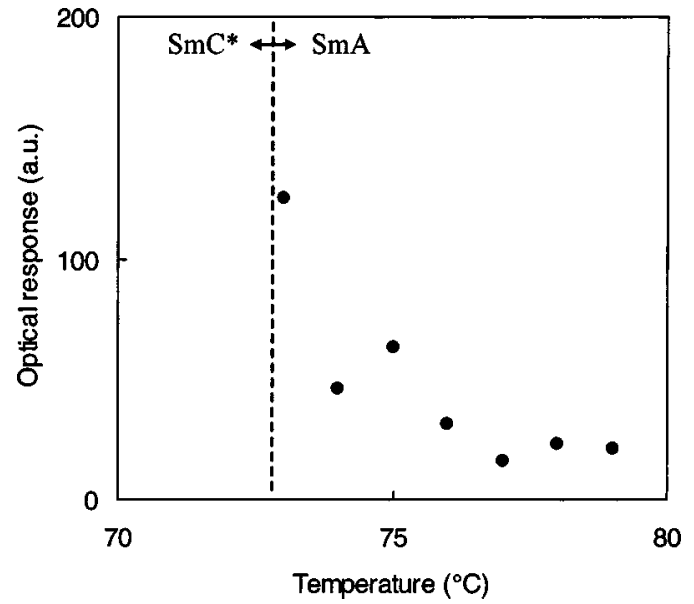

FIG. 8. Temperature dependence of the vibration intensity in the SmA phase of CS-1029.

molecules moved perpendicular to the electric field.

In the SmA phase, the molecules tilt with respect to the layer normal upon the field application, which is the socalled electroclinic effect. However, the field-induced tilt in the SmA phase is restricted to the plane perpendicular to the field, which coincides with the incident plane of the light in the geometry used in this study. Therefore, the refractive index for the $s$ polarization does not change upon the field application and no reflectance change should be observed in the SmA phase because the reflectance change should be caused by the change in refractive index in the film. When the vibration of FSF is excited by the electric field, the reflected light beam is deflected with respect to the initial beam position without electric field application at which the detected signal is the highest. If the phase of the excited vibration of the FSF is the same as that of the applied electrical signal, the detected light intensity changes as the secondharmonic of the input signal.

Figure 8 shows the temperature dependence of the vibration intensity. In this measurement, CS-1029 (Chisso), which shows a simple $\mathrm{SmA}-\mathrm{SmC}^{*}$ phase transition and has no subphase between the SmA and $\mathrm{SmC}^{*}$ phases, was used. Thus, an increase of the vibration intensity near the phase transition point from the $\mathrm{SmA}$ phase to the $\mathrm{SmC}^{*}$ phase was observed. This indicates that the electroclinic effect is related to the vibration in the SmA phase.

\section{B. SmC* phase}

In the $\mathrm{SmC}^{*}$ phase, the optical response of the reflected light can be detected even in the open-aperture geometry in contrast to the case in the SmA phase. This indicates that not only the deflection but also other origins of the optical response should be taken into account, namely, the change in the reflectance of the FSF and the scattering should be discussed in the interpretation of the multicomponents in the FFT spectrum of the reflected light. In the $\mathrm{SmC}^{*}$ phase, the transmitted light through a planar aligned sandwich cell is scattered immediately, after the polarity of the applied voltage is reversed, resulting in the transient decrease in the transmission light intensity. This phenomenon is called the 

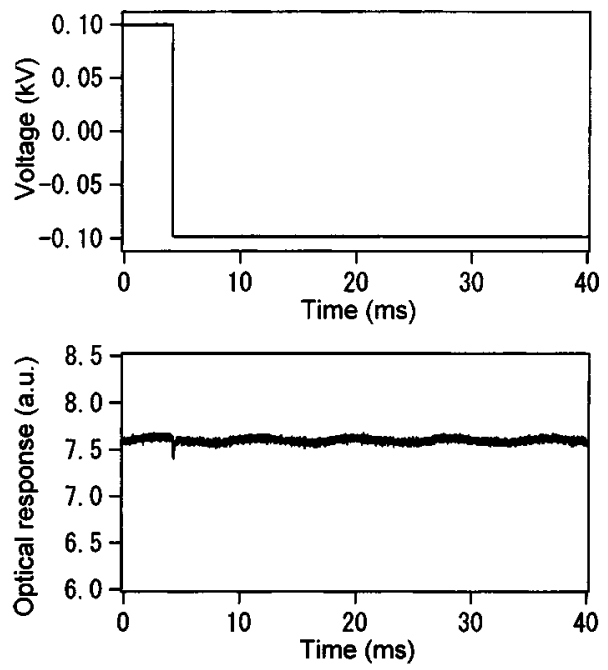

(a)
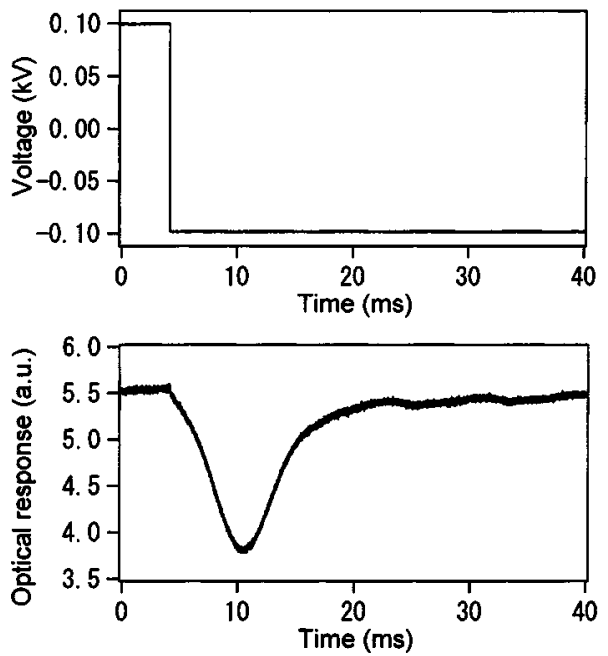

(b)

FIG. 9. Reflected light change in the open-aperture geometry upon polarity reversal of the applied voltage of MHPOBC; (a) SmA and (b) SmC*.

transient light scattering mode (TSM) and was reported by Yoshino and Ozaki. ${ }^{13}$ Figures 9(a) and 9(b) show the reflected light change in the open-aperture geometry upon the polarity reversal of the applied voltage in the SmA and SmC* phases, respectively. In the SmA phase, no optical response is observed, while in the $\mathrm{SmC}^{*}$ phase, the transient decrease in the reflected light at the FSF is observed. Therefore, even for the reflected light at the FSF, the TSM electrooptic effect is detectable. It should be noted that the wave form of the optical response strongly depends on the frequency of the applied alternating electric field.

Figure 10 shows the TSM response to the rectangular voltage in the FSF of MHPOBC as a function of frequency. At low frequency, a clear transient light decrease can be observed. At higher frequency, however, the transient light scattering is not settled in the period of the polarity reversals of the field. At $1 \mathrm{kHz}$, the optical response is strongly deformed, and the interval of the optical response becomes twice the period of the polarity reversal. In other words, the fundamental component of the optical response is observed.

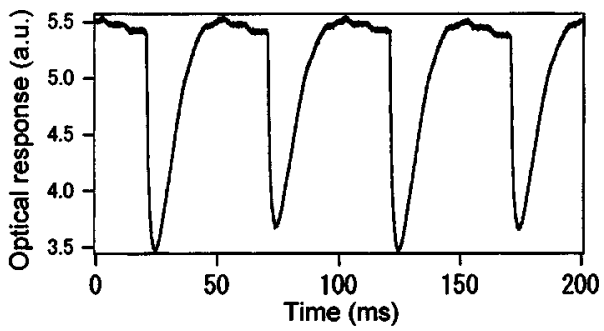

(a)

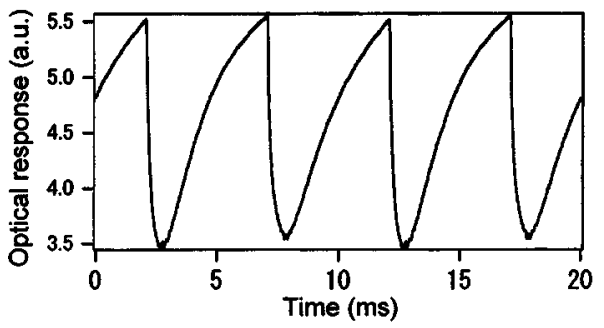

(b)

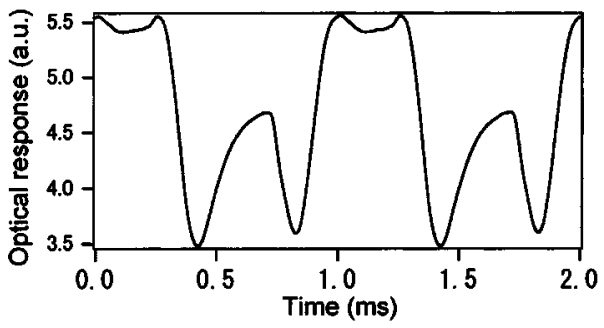

(c)

FIG. 10. TSM response to the rectangular voltage in the FSF of MHPOBC as a function of the frequency; (a) $10 \mathrm{~Hz}$, (b) $100 \mathrm{~Hz}$, and (c) $1 \mathrm{kHz}$.

In addition, as shown in Fig. 10(c), the rectangular-like wave form of the optical response is generated at high frequency. The FFT signal of such a rectangular wave form should be included as the odd component in the spectrum.

Figure 11 shows the optical response to the sinusoidal wave form of the applied voltage in the open-aperture geometry of MHPOBC at $1 \mathrm{~Hz}$ and $1 \mathrm{kHz}$. At $1 \mathrm{~Hz}$, the secondharmonic signal of the applied voltage appears due to the TSM effect immediately after the polarity reversal. At $1 \mathrm{kHz}$, however, the wave form of the optical response is deformed and has the same periodicity as the applied voltage, namely, the fundamental component is observed.

\section{C. $\mathrm{SmC}_{\mathrm{A}}^{*}$ phase}

Figure 12 shows the electric field dependence of the signal intensity of each component in the FFT spectra in the $\mathrm{SmC}_{\mathrm{A}}^{*}$ phase. The inclination of the second-harmonic component due to the film vibration increases at about $50 \mathrm{kV} / \mathrm{cm}$, and higher-order components also begin to be observed, similarly to the $\mathrm{SmC}^{*}$ phase. Although the reason for the appearance of the fundamental component in the small electric field is not clear, the fundamental component also began to increase at about $50 \mathrm{kV} / \mathrm{cm}$. It is considered that the antiferroelectric-ferroelectric phase transition occurred near $50 \mathrm{kV} / \mathrm{cm}$. 


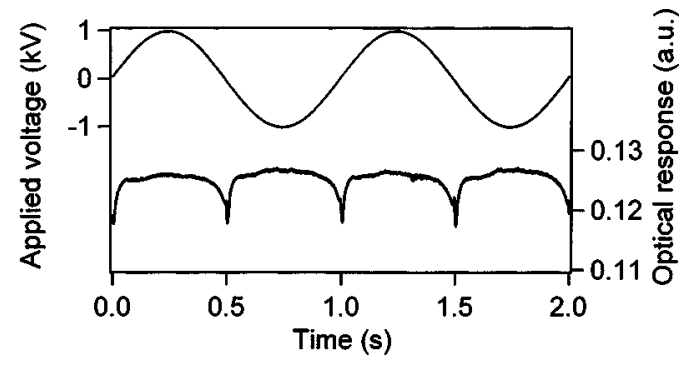

(a)

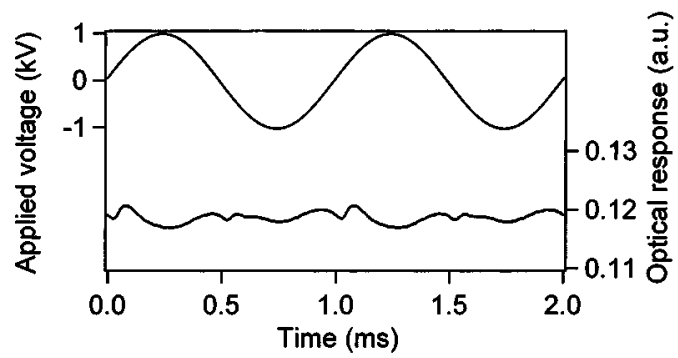

(b)

FIG. 11. Optical response to the sinusoidal wave form of the applied voltage in an open-aperture geometry in the $\mathrm{SmC}^{*}$ phase of MHPOBC; (a) $1 \mathrm{~Hz}$ and (b) $1 \mathrm{kHz}$.

\section{SUMMARY}

The conclusions of this study are summarized as follows. The electro-optical response of the reflected light on the surface of the FSF in the $\mathrm{SmA}, \mathrm{SmC}^{*}$, and $\mathrm{SmC}_{\mathrm{A}}^{*}$ phases was studied. Film vibration was generated by the electric field and was observed as a second-harmonic component of the FFT spectrum.

In the SmA phase, only the second-harmonic component appeared in the FFT spectra of the reflected light, and the molecular movement perpendicular to the electric field and the increase of the vibration near the phase transition point to the $\mathrm{SmC}^{*}$ phase, which are considered to be based on the electroclinic effect, were observed.

In the $\mathrm{SmC}^{*}$ phase, however, many other components also appeared in the FFT spectra of the reflected light. These complicated characteristics observed in the ferroelectric phase might be attributed to the TSM.

On the other hand, in the $\mathrm{SmC}_{\mathrm{A}}^{*}$ phase, only the secondharmonic component appeared, similar to the case of the SmA phase. In the electric field high enough to cause an antiferroelectric-ferroelectric phase transition, however, many other components appear in the spectrum, in the same manner as that in the $\mathrm{SmC}^{*}$ phase.

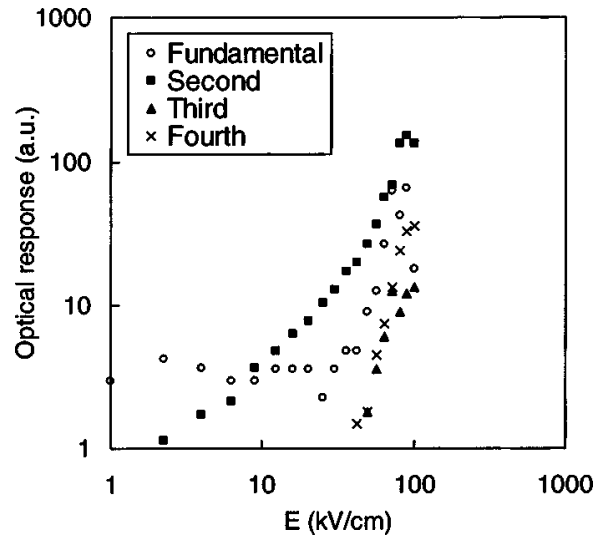

FIG. 12. Electric field dependence of the signal intensity of each component in the FFT spectra in the $\mathrm{SmC}_{\mathrm{A}}^{*}$ phase of MHPOBC.

\section{ACKNOWLEDGMENT}

We would like to show our acknowledgment to Chisso for providing MHPOBC. This work is supported by a Grantin-Aid for Scientific Research from the Japan Ministry of Education, Culture, Sports, Science, and Technology (14350165).

${ }^{1}$ C. Rosenblatt, R. Pindak, N. A. Clark, and R. B. Meyer, Phys. Rev. Lett. 42, 1220 (1979).

${ }^{2}$ S. Heinekamp, R. A. Pelcovits, E. Fontes, E. Y. Chen, R. Pindak, and R. B. Meyer, Phys. Rev. Lett. 51, 1017 (1984).

${ }^{3}$ I. Kraus, P. Pieranski, E. Demikhov, H. Stegemeyer, and J. Goodby, Phys. Rev. E 48, 1916 (1993).

${ }^{4}$ E. I. Demikhov, E. Hoffmann, H. Stegemeyer, S. A. Pikin, and A. Strigazzi, Phys. Rev. E 51, 5954 (1995).

${ }^{5}$ S. Uto, H. Ohtsuki, M. Ozaki, and K. Yoshino, Appl. Phys. Lett. 69, 1503 (1996).

${ }^{6}$ S. Uto, H. Ohtsuki, M. Ozaki, and K. Yoshino, Jpn. J. Appl. Phys., Part 1 35, 5050 (1996).

${ }^{7}$ S. Uto, E. Tazoh, M. Ozaki, and K. Yoshino, Jpn. J. Appl. Phys., Part 2 36, L1198 (1997).

${ }^{8}$ S. Uto, S. Okazaki, M. Ozaki, and K. Yoshino, J. Soc. Electron. Mater. Eng. 5, 22 (1996).

${ }^{9}$ A. Jakli, L. Bata, A. Buka, and N. Eber, Ferroelectrics 69, 153 (1986).

${ }^{10}$ S. V. Yablonskii, T. Oue, H. Nambu, A. S. Mikhailov, M. Ozaki, and K. Yoshino, Appl. Phys. Lett. 75, 64 (1999).

${ }^{11}$ S. V. Yablonskii, K. Nakano, A. S. Mikhailov, M. Ozaki, and K. Yoshino, Appl. Phys. Lett. 80, 571 (2002).

${ }^{12}$ P. Pieranski, L. Beliard, J.-Ph. Tournellec, X. Leoncini, C. Furtlehner, H. Dumoulin, E. Riou, B. Jouvin, J.-P. Fenerol, Ph. Palaric, J. Heuving, B. Cartier, and I. Kraus, Physica A 194, 364 (1993).

${ }^{13}$ K. Yoshino and M. Ozaki, Jpn. J. Appl. Phys., Part 2 23, L385 (1984). 